January 2009

\title{
Nurses attitudes towards perinatal bereavement care
}

Chan Moon Fai

National University of Singapore, Singapore

David Arthur

Aga Khan University, david.arthur@aku.edu

Follow this and additional works at: https://ecommons.aku.edu/pakistan_fhs_son

Part of the Nursing Midwifery Commons

\section{Recommended Citation}

Moon Fai, C., Arthur, D. (2009). Nurses attitudes towards perinatal bereavement care. Journal of Advanced Nursing, 65(12), 2532-2541.

Available at: https://ecommons.aku.edu/pakistan_fhs_son/218 


\title{
ORIGINAL RESEARCH
}

\section{Nurses' attitudes towards perinatal bereavement care}

\author{
Moon Fai Chan \& David Gordon Arthur
}

Accepted for publication 10 July 2009

Correspondence to: M. F. Chan:

e-mail: nurcmf@nus.edu.sg

Moon Fai Chan PhD CStat

Assistant Professor

Alice Lee Centre for Nursing Studies, Yong

Loo Lin School of Medicine, National

University of Singapore, Singapore

David Gordon Arthur PhD RN

Professor

Alice Lee Centre for Nursing Studies, Yong

Loo Lin School of Medicine, National

University of Singapore, Singapore

CHAN M.F. \& ARTHUR D.G. (2009) Nurses' attitudes towards perinatal bereavement care. Journal of Advanced Nursing 65(12), 2532-2541.

doi: 10.1111/j.1365-2648.2009.05141.x

\begin{abstract}
Title. Nurses' attitudes towards perinatal bereavement care.

Aim. This paper is a report of a study conducted to explore the factors associated with nurses and midwives' attitudes towards perinatal bereavement care.

Background. Caring for and supporting parents whose infant has died is extremely demanding, difficult and stressful. In some situations nurses may experience personal failure, feel helpless, and need to distance themselves from bereaved parents because they feel unable to deal with the enormity of the parental feelings of loss.

Method. A correlational questionnaire study using convenience sampling was carried out in Singapore in 2007 with 185 nurses/midwives in one obstetrics and gynaecology unit.

Results. Regression models showed that nurses/midwives with religious beliefs and those with more positive attitudes to the importance of hospital policy and training for bereavement care were statistically significantly more likely to have a positive attitude towards perinatal bereavement care. Nurses emphasized their need for increased knowledge and training on how to cope with bereaved parents and requested greater support from team members and the hospital.

Conclusion. Bereavement counselling education and preceptorship supervision are recommended to reduce this stressful experience, increase the confidence and expertise of novices, and lead to increased quality of care for bereaved parents.
\end{abstract}

Keywords: attitudes, hospital policy, midwives, nurses, perinatal bereavement care, religion

\section{Introduction}

Perinatal loss is generally regarded as the most painful form of bereavement because it is unexpected, often sudden and sometimes unexplained (Chambers \& Chan 2000). The general public often dismisses perinatal loss as not being true bereavement, and its traumatic potential has long been overlooked by clinicians and researchers [Stillbirth and
Neonatal Death Society (SANDS) 1991]. Psychiatric morbidity such as depression, anxiety disorders, and somatic symptoms has been closely associated with such loss (Hughes et al. 1999; Säflund et al. 2004). In addition, feelings of helplessness are common, and bereaved parents need care and support through sensitive handling and careful communication (Hughes et al. 1999; Gaze 2000). SANDS recommends that all community health practitioners supporting bereaved 
parents should have access to basic, postbasic and in-service training to equip them to offer adequate care to such families (SANDS 1991). For many bereaved parents, the care that nursing staff provide may have a crucial effect on their response to such a death (Engler \& Lasker 2000; RowaDewar 2002). However, caring for and supporting parents whose infant has died is extremely demanding, difficult and stressful (Gensch \& Midland 2000; Säflund et al. 2004), and in some situations nursing staff may experience personal failure because they feel helpless and unable to provide help (Robinson et al. 1999). Some nurses have commented that they need to distance themselves from bereaved parents because they feel unable to deal with the enormity of the parental feelings of loss (Paterson \& Zderad 1988; Säflund et al. 2004). In addition, language or cultural barriers influence nurses' involvement in bereavement care (Engler et al. 2004).

\section{Background}

Nurses and midwives are often relied on to care for bereaved parents and families affected by these traumatic events, which are stressful for all involved (Black \& Tufnell 2006), and are expected to interact with the bereaved in a supportive manner, regardless of whether they feel adequately prepared or disposed to do so (Cox \& Briggs 2004). The theory of culture care diversity and universality was developed by Leininger (1997), and she emphasized that nurses should expand their thinking from a unicultural perspective to one that was multicultural, holistic, and comparative. Hence, because of the increasing need to work with families of divergent cultures, it is important for nurses to be familiar with the traditions and beliefs of others as well as what loss means to different individuals (Speck 1978; Gibson 1998). One of the culturally specific communication practices recommended for nurses when caring for bereaved family who are from Asia is speaking to the bereaved parent through a 'key person' (Engler et al. 2004). This person could be a respected or older person in the bereaved family. This, in turn, helps to develop positive attitudes; this can affect the quality of care provided (Gaze 2000; Birtwistle et al. 2002), for example by helping bereaved couples to cope with the grieving process and create healing memories.

In Asian countries, people treat death as a taboo subject (Yam et al. 2001; Ping et al. 2002), and attitudes towards bereavement are strongly influenced by the principles of Confucianism and Buddhism, which teach the importance, especially amongst men, of not crying or expressing negative emotion outside the family (Martinson 1998; Ping et al. 2002). The bereaved person often feels ashamed to show grief, especially outside their family (Wu \& Tseng 1985). Yet the pain of losing a young child is revealed in the Chinese saying 'The white head witnesses the death of the black head with great pain' (the older generation witnesses the death of the younger generation), and for those with strong traditional beliefs, the pain is compounded by their need to be stoic and not reveal their true feelings at the loss of an infant. For bereaved couples, this very often means avoiding any discussion of the death to 'protect' relatives such as their parents or siblings (Gao et al. 1996). In the family, the centre of Singapore culture, the wishes of the bereaved couple must be respected. As the family interaction pattern is determined by the hierarchical family structure, nurses have to be very prudent in their approach to minimize any extreme expression of public emotion and to avoid situations that may imperil the parents' relationship. Since Singaporeans often avoid open displays of emotion or discussion of their feelings, especially with people outside the family, nurses must be able to pick up on the subtle and non-verbal ways of expressing emotion (Yam et al. 2001; Ping et al. 2002). These issues complicate bereavement care and make it difficult for affected parents to seek help. Furthermore, studies have shown that Asian people very often present emotional problems as somatic complaints (Wu \& Tseng 1985). As a result of these cultural values or traits, it may not be easy for bereaved parents to express their needs nor for nurses or midwives to provide adequate help in the bereavement process.

In the past 10 years, some hospitals in Singapore have developed perinatal bereavement support groups in which staff educate, counsel and support bereaved parents. The role of these groups is to offer support and care to bereaved couples and to facilitate their recovery as they go through the grieving process. However, previous studies have revealed that hospital-based bereavement support services focus mainly on sorrow and harm reduction for bereaved families but seldom explore the needs of healthcare professionals (Chan \& Chow 1998; Chan et al. 2005).

In 2004, the Child Bereavement Support (CBS) (Singapore) (http://www.childbereavementsupport.org.sg/; http://www. childbereavementsupport.org.sg/resources.html) was formed by a group of bereaved parents and healthcare professionals to support bereaved parents and to educate healthcare professionals (CBS; http://www.childbereavementsupport. org.sg/). Their first book, Farewell, My Child, published in 2005 , is a collection of stories of childrens' deaths with examples of how the parents coped, and it explains how giving support to each other is therapeutic. Other related publications have addressed personal stories, shared experiences, and given advice or support for bereaved families, but few have examined nurses and midwives' needs and the supports 
necessary while providing perinatal bereavement care (CBS; http://www.childbereavementsupport.org.sg/resources.html).

Singaporean nurses/midwives work in a multi-cultural environment and are expected to understand the needs of Chinese, Malay and Indian patients, yet most of the literature is Eurocentric. To help nurses and midwives care for and support parents whose baby has died, and cope with their own feelings in this extremely demanding, difficult and stressful time, it is recommended that basic, postbasic and in-service education be provided (Gensch \& Midland 2000). Special skills are needed to help bereaved parents, and after appropriate training it is logical to assume that nurses and midwives are better equipped to cope with perinatal bereavement (SANDS 1991). Typically, nurses receive little education to prepare them for dealing with the death of an infant or for assisting bereaved couples during and after this time (White et al. 2001; Engler et al. 2004). Szgalsky (1989) and Rybarik (1996) agreed that supportive and educational measures were necessary to help nurses in their work with bereaved people.

More recently, in a study in United States of America, Engler et al. (2004) found that nurses who had more experience and education related to bereavement were more comfortable providing this care. In addition, they found that language or cultural differences are influential factors in the level of nurses' involvement with bereaved families (Engler et al. 2004). In a study in Hong Kong, Yam et al. (2001) found that nurses felt ambivalent, helpless, and lacking knowledge and counselling skills in caring for dying infants and their bereaved families. In another study in Hong Kong, Chan et al. (2004) found that nurses' attitudes towards bereavement care were positively correlated with needs for training in bereavement care and support via hospital policies.

Hence, nursing models emphasize the importance of the assessment of bereaved families in a culturally sensitive manner and assist caregivers in increasing their effectiveness, overcoming cultural ignorance and coping better with their own grief responses (Leininger 1997; Keeney 2004). Much research has been conducted to investigate the needs and feelings of bereaved families and the care practices that are helpful to them, but there little information is available about the experience of, and factors affecting nurses' attitudes towards perinatal bereavement care.

\section{The study}

\section{Aim}

The aim of the study was to explore the factors associated with nurses' and midwives' attitudes towards perinatal bereavement care. We addressed two research questions: (1) What are nurses and midwives' attitudes towards perinatal bereavement care? and (2) What factors are associated with nurses and midwives' attitudes towards bereavement care?

\section{Design}

This study was a correlational study. The power analysis of the study was based on the attitudes towards perinatal bereavement care. A multiple regression model was used and 4-5 covariates were expected in the model (Chan et al. 2004). With an expected squared multiple correlation of $0 \cdot 325$, a sample size of 180 was required for the study to achieve $80 \%$ power at the $5 \%$ significance level (nQuery Advisor 2001).

\section{Participants}

Convenience sampling was chosen, and involved all nurses and midwives working in one obstetrics and gynaecology $(\mathrm{O} \& \mathrm{G})$ unit in Singapore. The nurses working in the unit hold Enrolled or Registered Nurse qualifications and also a midwifery qualification.

\section{Data collection}

The data were collected in 2007 using a structured selfreport questionnaire. The questionnaire, was developed by Chan et al. (2004), proved valid and reliable in a study with Chinese nurses in Hong Kong. The instrument has three sections. Section 1 collects demographic data (i.e. age, education level, recent ranking and religious background), experience (i.e. personal grieving, handling grieving clients and years of work in the obstetrics and gynaecology unit) and training (i.e. midwifery and bereavement care). In the original version, section 2 comprised eight attitude statements formulated to measure nurses' attitudes towards bereavement care (factor 1), but two questions were removed because of their low factor loadings (0.24), leaving a total of six questions for this study, and a high score indicates a positive and favourable attitude towards perinatal bereavement care. Section 3 was composed of two parts; the first comprised three statements used to evaluate nurses' attitudes towards the importance of hospital policy on perinatal bereavement (factor 2), with a high score indicating attitudes that are highly influenced by policy and a high demand for nurses with perinatal bereavement care training. The second part comprised four statements to evaluate nurses' attitudes towards the 
importance of formal training to deal with perinatal bereavement care (factor 3). In section 3, nurses were asked to rank each item, in each section, on 6-point Likerttype scales: (0) Not applicable, (1) Very unimportant or completely disagree, to (5) Very important or completely agree. Exploratory factor analysis (Table 1) was conducted to establish the construct validity of the instrument and three factors were emerged that accounted for $72.4 \%$ of the variance, with $52.3 \%, 11.9 \%$, and $8.2 \%$ for factors 1 , 2 , and 3 respectively $\left(\mathrm{KMO}=0.89, \quad \chi^{2}=1618 \cdot 2\right.$, $P<0.001)$. Cronbach's alpha values for factor 1,2 , and 3 were $0.85,0.92$ and 0.92 respectively.

Ward managers were asked to deliver information sheets, consent forms and questionnaire to all nurses and midwives in their ward to explain the aim of the study. Participants were asked to complete and return the consent forms and anonymous questionnaires within 2 weeks for collection from two boxes placed in each unit by the research team. These procedures minimized the possibility of ward managers knowing who had or had not participated in the study.

\section{Ethical considerations}

The study was approved by the appropriate ethics committee.

\section{Data analysis}

Exploratory factor analysis was used to identify the factorial construct of the instrument, and Cronbach's alpha coefficients were used to examine the internal reliability of the factor structure, while descriptive statistics were used to examine participants' background variables. A normality test revealed that the attitude scores were not normally distributed therefore nonparametric tests were used for further analysis. Although most of the data were not normally distributed, but our data set was large $(n=185)$ enough to presume that the data were normal. This assumption was based on the foundation of the central limit theorem that the cumulative distribution function of a variable will tends to be normal if its size was large (Spiegel 1992).

To examine which factors contributed to the attitude scores, bivariate analyses including Mann-Whitney $U$ and

Table 1 Factor analysis of responses $(n=185)$ and comparison of responses on each item

\begin{tabular}{|c|c|c|c|c|c|}
\hline \multirow[b]{2}{*}{ Factor } & \multirow{2}{*}{$\begin{array}{l}\text { Factor } \\
\text { loading }\end{array}$} & \multirow[b]{2}{*}{ Cronbach's $\alpha^{*}$} & \multicolumn{3}{|l|}{ Score } \\
\hline & & & $1-2, n(\%)$ & $3, n(\%)$ & $4-5, n(\%)$ \\
\hline \multicolumn{6}{|l|}{ Factor 1: attitudes to perinatal bereavement care ${ }^{\dagger}$} \\
\hline $\begin{array}{l}\text { 1. I believe that the Grief Caring Programme can provide } \\
\text { psychological support to the bereaved couple }\end{array}$ & $0 \cdot 60$ & $0 \cdot 85$ & $2(1 \cdot 1)$ & $11(5 \cdot 9)$ & $172(93 \cdot 0)$ \\
\hline $\begin{array}{l}\text { 2. I agree that the 'Good-bye Baby' Parent Support Group } \\
\text { provides support to parents with similar experiences }\end{array}$ & $0 \cdot 54$ & & $3(1 \cdot 6)$ & $18(9 \cdot 7)$ & $164(88 \cdot 6)$ \\
\hline 3. I respect the bereaved parent's feelings and needs & $0 \cdot 81$ & & $0(0 \cdot 0)$ & $3(1 \cdot 6)$ & $181(98 \cdot 4)$ \\
\hline $\begin{array}{l}\text { 4. I communicate with parents in a clear, sensitive and } \\
\text { honest manner }\end{array}$ & $0 \cdot 77$ & & $1(0 \cdot 5)$ & $13(7 \cdot 1)$ & $169(92 \cdot 3)$ \\
\hline 5. I agree that parents should be given time to grieve & $0 \cdot 84$ & & $1(0 \cdot 5)$ & $2(1 \cdot 1)$ & $182(98 \cdot 4)$ \\
\hline $\begin{array}{l}\text { 6. All those involved in the care of bereaved parents should } \\
\text { be well-informed }\end{array}$ & $0 \cdot 69$ & & $3(1 \cdot 6)$ & $8(4 \cdot 3)$ & $173(94 \cdot 0)$ \\
\hline \multicolumn{6}{|c|}{ Factor 2: attitudes on the importance of hospital policy to bereavement care } \\
\hline $\begin{array}{l}\text { 7. The unit should have a clear policy for the management } \\
\text { of bereavement }\end{array}$ & $0 \cdot 82$ & $0 \cdot 92$ & $2(1 \cdot 1)$ & $27(14 \cdot 8)$ & $153(84 \cdot 1)$ \\
\hline $\begin{array}{l}\text { 8. All staff involved should be well-informed about the } \\
\text { policy }\end{array}$ & $0 \cdot 85$ & & $2(1 \cdot 1)$ & $15(8 \cdot 2)$ & $167(90 \cdot 8)$ \\
\hline 9. All staff involved should understand the policy & $0 \cdot 87$ & & $1(0 \cdot 5)$ & $15(8 \cdot 2)$ & $167(91 \cdot 3)$ \\
\hline \multicolumn{6}{|l|}{ Factor 3: attitudes to training for bereavement care ${ }^{\ddagger}$} \\
\hline 10. Joining a training programme on bereavement care & $0 \cdot 78$ & $0 \cdot 92$ & $4(2 \cdot 2)$ & $26(14 \cdot 1)$ & $154(83 \cdot 7)$ \\
\hline 11. Participating in bereavement care & $0 \cdot 82$ & & $5(2 \cdot 7)$ & $30(16 \cdot 3)$ & $149(81 \cdot 0)$ \\
\hline $\begin{array}{l}\text { 12. Sharing experiences with colleagues and working as a } \\
\text { team }\end{array}$ & $0 \cdot 84$ & & $6(3 \cdot 3)$ & $26(14 \cdot 2)$ & $151(82 \cdot 5)$ \\
\hline 13. Seeking support when experiencing 'burnout' & $0 \cdot 75$ & & $5(2 \cdot 7)$ & $17(9 \cdot 2)$ & $162(88 \cdot 0)$ \\
\hline
\end{tabular}

*Cronbach's alpha.

${ }^{\dagger}$ Strongly agree (5), agree (4), uncertain (3), disagree (2), strongly disagree (1), not applicable (0).

tVery important (5), important (4), uncertain (3), unimportant (2), very unimportant (1), not applicable (0). 
Kruskal-Wills tests were used, while Spearman's correlation coefficient $\left(r_{s}\right)$ was used to examine the relationships among nurses' attitudes towards bereavement care, importance of hospital policy to bereavement care, and training for bereavement care. Although most of the data were not normally distributed, our data set was large $(n=185)$ enough to presume that the data were normal. This assumption was based on the central limit theorem that the cumulative distribution function of a variable will tend to be normal if its size is large, e.g. $n>100$. (Spiegel 1992).

A multiple regression (stepwise) model was used to identify which factors predicted attitude scores towards perinatal bereavement care, with the level of statistical significance set at $P<0.05$.

\section{Results}

\section{Participant demographics}

Overall, 276 questionnaires were distributed and 185 (67\% response rate) were returned within 2 weeks. Table 2 shows that there were 19 senior nurses/midwives $(10 \cdot 3 \%)$ and 160 Enrolled/Registered Nurse/Midwives (87.0\%). More than $42.0 \%(n=77)$ were aged 41 years or above, $75.5 \%$ $(n=138)$ had a religious background and more than $53.0 \%$ $(n=98)$ had more than 11 years' experience in the O\&G unit. Only $24 \cdot 4 \% \quad(n=44)$ held a Bachelor's degree in nursing, while the remainder were diploma or certificate holders. There were $61 \cdot 4 \%(n=113)$ with experience in handling grieving clients, but $<25 \%(n=47)$ had taken a bereavement care course.

\section{Attitudes towards perinatal bereavement care}

Table 1 displays the frequencies of responses to items on attitudes towards bereavement care (statements 1-6). Most $(n=172,93.0 \%)$ strongly agreed/agreed that a grief counselling programme can provide psychological support for the bereaved couple, $98.4 \%(n=182)$ strongly agreed/ agreed that bereaved parents should be given time to grieve, $94.0 \% \quad(n=173)$ strongly agreed/agreed that all those involved in the care of bereaved parents should be well-informed, and $92.3 \% \quad(n=169)$ that staff should communicate with bereaved parents in a clear, sensitive and honest manner. However, $11 \cdot 3 \%(n=21)$ disagreed/ were uncertain that the 'Good-bye Baby' Parent Support Group provides support for parents with such an experience, and $5.9 \%(n=11)$ disagreed/were uncertain that all involved in the care of bereaved parents should be wellinformed. The results, in general, showed that the majority had indicated a positive attitude towards perinatal bereavement care.

Three items (statements 7-9) were related to hospital policy support for bereavement practices (see Table 1) and $84 \cdot 1 \%$ of respondents $(n=153)$ claimed that it is very important/important that there should be a clear policy for the management of bereavement care in the obstetric unit. Over $90 \%$ claimed that it was very important/important that

Table 2 Participants demographics

\begin{tabular}{|c|c|}
\hline Demographic & $\begin{array}{l}\text { Total }(n=185), \\
n(\%)\end{array}$ \\
\hline \multicolumn{2}{|l|}{ Age (years)* } \\
\hline $18-25$ & $24(13 \cdot 3)$ \\
\hline $26-30$ & $28(15 \cdot 5)$ \\
\hline $31-35$ & $14(7 \cdot 7)$ \\
\hline $36-40$ & $38(21 \cdot 0)$ \\
\hline $41+$ & $77(42 \cdot 5)$ \\
\hline \multicolumn{2}{|l|}{ 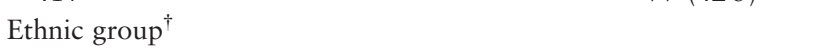 } \\
\hline Chinese & $90(49 \cdot 1)$ \\
\hline Malay & $44(24 \cdot 0)$ \\
\hline Indian & $35(19 \cdot 1)$ \\
\hline Other (e.g. European, American) & $14(7 \cdot 7)$ \\
\hline \multicolumn{2}{|l|}{ Staff grade } \\
\hline Nursing/midwifery student & $5(2 \cdot 7)$ \\
\hline Enrolled Nurse/Registered Nurse/Midwife & $160(87 \cdot 0)$ \\
\hline Senior nurse/midwife & $19(10 \cdot 3)$ \\
\hline \multicolumn{2}{|l|}{ Educational level ${ }^{\S}$} \\
\hline Diploma or below & $136(75 \cdot 6)$ \\
\hline Bachelor or above & $44(24 \cdot 4)$ \\
\hline \multicolumn{2}{|l|}{ Experience in obstetrics and gynaecology (years) } \\
\hline$<5$ & $48(26 \cdot 1)$ \\
\hline $5-10$ & $38(20 \cdot 7)$ \\
\hline $11+$ & $98(53 \cdot 3)$ \\
\hline \multicolumn{2}{|l|}{ Midwifery training $^{\dagger}$} \\
\hline Yes & $84(45 \cdot 9)$ \\
\hline No & $99(54 \cdot 1)$ \\
\hline \multicolumn{2}{|l|}{ Religious beliefs ${ }^{\dagger}$} \\
\hline Yes & $138(75 \cdot 5)$ \\
\hline No & $46(25 \cdot 0)$ \\
\hline \multicolumn{2}{|l|}{ Personal grieving experiences ${ }^{\dagger}$} \\
\hline Yes & $116(63 \cdot 4)$ \\
\hline No & $67(36 \cdot 6)$ \\
\hline \multicolumn{2}{|c|}{ Taken training/courses related to bereavement care* } \\
\hline Yes & $47(25 \cdot 5)$ \\
\hline No & $137(74 \cdot 5)$ \\
\hline \multicolumn{2}{|l|}{ Past experience in handling grieving parents ${ }^{\ddagger}$} \\
\hline Yes & $113(61 \cdot 4)$ \\
\hline No & $71(38 \cdot 6)$ \\
\hline
\end{tabular}


they should be well-informed $(n=167)$ and the policy understood by all staff $(n=167)$.

Four items (statements 10-13) reflected the need for formal education in grief counselling (see Table 1). More than $82 \%$ percent said that they would share experiences $(n=151)$ with their colleagues and seek support when feeling 'burnout' $(n=162)$, while $83 \cdot 7 \% \quad(n=154)$ replied that it was very important/important to have opportunities to join a training programme on bereavement care.

\section{Factors associated with attitudes towards perinatal bereavement care}

To determine which factors might be associated with respondents' attitudes towards bereavement care, a multi-

Table 3 Univariate comparison of attitudes towards bereavement care scores by demographics

\begin{tabular}{|c|c|c|c|c|}
\hline Demographic & $n$ & Mean \pm SD & Statistics & $P$ value \\
\hline \multicolumn{5}{|l|}{ Age $(\text { years })^{\dagger}$} \\
\hline$\leq 25$ & 24 & $26 \cdot 0 \pm 2 \cdot 8$ & $11 \cdot 2$ & $0.025 \%$ \\
\hline $26-30$ & 28 & $25 \cdot 9 \pm 3 \cdot 2$ & & \\
\hline $31-35$ & 14 & $28 \cdot 1 \pm 2 \cdot 0$ & & \\
\hline $36-40$ & 38 & $27 \cdot 5 \pm 3 \cdot 1$ & & \\
\hline$>41$ & 77 & $26 \cdot 9 \pm 3 \cdot 5$ & & \\
\hline \multicolumn{5}{|l|}{ Staff grade $^{\dagger}$} \\
\hline Nursing/midwifery student & 6 & $26 \cdot 8 \pm 2 \cdot 5$ & $8 \cdot 8$ & $0 \cdot 008 * *$ \\
\hline Enrolled Nurse/Registered Nurse/Registered Midwife & 291 & $26 \cdot 5 \pm 2 \cdot 4$ & & \\
\hline Senior nurse/midwife & 26 & $28 \cdot 0 \pm 2 \cdot 2$ & & \\
\hline \multicolumn{5}{|l|}{ Ethnic group $^{\dagger}$} \\
\hline Chinese & 90 & $26 \cdot 5 \pm 3 \cdot 0$ & $5 \cdot 0$ & $0 \cdot 169$ \\
\hline Malay & 44 & $26 \cdot 7 \pm 4 \cdot 2$ & & \\
\hline Indian & 35 & $27 \cdot 3 \pm 2 \cdot 4$ & & \\
\hline Other (e.g. European, American) & 14 & $28 \cdot 3 \pm 2 \cdot 1$ & & \\
\hline \multicolumn{5}{|l|}{ Education level $^{\ddagger}$} \\
\hline Diploma or below & 136 & $26 \cdot 7 \pm 2 \cdot 9$ & $2626 \cdot 5$ & $0 \cdot 218$ \\
\hline Bachelor or above & 44 & $26 \cdot 9 \pm 4 \cdot 2$ & & \\
\hline \multicolumn{5}{|l|}{ Experience in obstetrics and gynaecology (years) ${ }^{\dagger}$} \\
\hline$<5$ & 48 & $26 \cdot 6 \pm 2 \cdot 8$ & $8 \cdot 61$ & $0 \cdot 014 *$ \\
\hline $5-10$ & 38 & $25 \cdot 9 \pm 3 \cdot 1$ & & \\
\hline $11+$ & 98 & $27 \cdot 3 \pm 3 \cdot 4$ & & \\
\hline \multicolumn{5}{|l|}{ Midwifery training ${ }^{\ddagger}$} \\
\hline Yes & 84 & $27 \cdot 3 \pm 2 \cdot 8$ & $3680 \cdot 5$ & $0 \cdot 175$ \\
\hline No & 99 & $26 \cdot 5 \pm 3 \cdot 5$ & & \\
\hline \multicolumn{5}{|l|}{ Religious beliefs ${ }^{\star}$} \\
\hline Yes & 138 & $27 \cdot 2 \pm 3 \cdot 2$ & $2271 \cdot 0$ & $0.003 * *$ \\
\hline No & 46 & $25 \cdot 8 \pm 3 \cdot 1$ & & \\
\hline \multicolumn{5}{|l|}{ Personal grieving experiences ${ }^{\ddagger}$} \\
\hline Yes & 116 & $27 \cdot 2 \pm 3 \cdot 3$ & $3089 \cdot 0$ & $0 \cdot 019 *$ \\
\hline No & 67 & $26 \cdot 3 \pm 3 \cdot 0$ & & \\
\hline \multicolumn{5}{|l|}{ Taken training/courses related to bereavement care } \\
\hline Yes & 47 & $27 \cdot 7 \pm 2 \cdot 4$ & $2573 \cdot 0$ & $0 \cdot 037^{*}$ \\
\hline No & 137 & $26 \cdot 6 \pm 3 \cdot 4$ & & \\
\hline \multicolumn{5}{|l|}{ Past experience in handling grieving parents ${ }^{\ddagger}$} \\
\hline Yes & 113 & $27 \cdot 3 \pm 3 \cdot 3$ & $2946 \cdot 5$ & $0 \cdot 002 \% *$ \\
\hline No & 71 & $26 \cdot 1 \pm 2 \cdot 9$ & & \\
\hline Attitudes on importance of hospital policy to bereavement care ${ }^{\mathbb{S}}, r_{s}$ & 184 & & $0 \cdot 56$ & $<0.001 * *$ \\
\hline Attitudes to training for bereavement care ${ }^{\S}, r_{s}$ & 184 & & $0 \cdot 59$ & $<0.001 * *$ \\
\hline
\end{tabular}

${ }^{\dagger}$ Kruskal-Wallis.

${ }^{\star}$ Mann-Whitney U-test.

${ }^{\$}$ Spearman coefficient.

Statistically significant at $* P<0.05 ; * P<0.01$; NA, not available. 
variate analysis was performed. First, a bivariate analysis (see Table 3) revealed statistically significant differences between the factors of age $(P=0.025)$, recent ranking $(P=0.008)$, experiences in O\&G $(P=0.014)$, religious beliefs $(P=0.003)$, personal grieving experiences $(P=0.019)$, having taken bereavement care training/courses $(P=0.037)$ and past experiences in handling grieving parents $(P=0.002)$ compared with attitude scores towards bereavement care. Statistically significant positive correlations were found between attitude scores towards bereavement care and attitudes towards the importance of hospital policy $(P<0.001)$ and training for bereavement care $(P<0.001)$.

Second, a multiple regression (stepwise) analysis was used to identify a model to predict attitude scores towards bereavement care (see Table 4). The results showed that those having religious orientation (Beta $=1 \cdot 12, P=0 \cdot 010$ ) and more positive attitudes towards the importance of hospital policy (Beta $=0.42, P<0.001)$ and training for bereavement care (Beta $=0.46, P<0.001)$ were statistically significantly more likely to have a positive attitude towards bereavement care. This model had a good fit $(F=41 \cdot 8$, $P<0 \cdot 001$ ), with an adjusted $R^{2}$ of $42 \cdot 0 \%$.

\section{Discussion}

While some important findings emerged which help inform future educational and clinical directions, some limitations should be mentioned First, the homogeneity of the sample introduces systematic bias as they were from one hospital, the majority were Chinese, and their educational level was diploma or below. Thus, generalization of the findings to the entire population of interest should be made with caution and future studies should be based on sufficient sample sizes from more hospitals. Further, based on our findings from the regression model, power analysis suggested that a sample size of approximately 366 was required to achieve $80 \%$ power and a $5 \%$ level of statistical significance. Second, we used a self-report questionnaire, which may have caused response bias (Polit \& Hungler 1995). For example, under some circumstances, socially desirable responses might result in responses appearing more normal or acceptable to the researcher. Third, bereaved parents were not surveyed, and so no comparisons between nurses' and parents' perceptions can be made. Finally, we recommend that a qualitative study using individual or focus group interviews should be carried out to explore the cultural subtleties of Singapore's three main cultural groups and the meaning of perinatal bereavement care for nurses.

This study paves the way for a more systematic study of perinatal bereavement care among nurses in Singapore as caring for bereaved parents clearly has a powerful impact. Our findings confirm the need for improved communication skills and greater support from team members. This is supported by previous researchers who have likewise suggested that psychological and emotional support for nurses is necessary to enable them to support bereaved parents (Gardner 1999; Birtwistle et al. 2002; Chan et al. 2004). Supportive care practices for nurses should be established, understood, and practised by all members of the perinatal care team (Beem et al. 1998; Birtwistle et al. 2002; Black \& Tufnell 2006).

In Singapore, three cultures (Chinese, Malay and Indian) involve many traditional rituals conducted during the grieving process that can facilitate the acceptance of death. In Singapore Chinese people, believe that the natural extension of a 'good death' connotes a combination of life accomplishments and death without suffering. In Chinese culture, death without suffering means death without pain or physical

\begin{tabular}{|c|c|c|c|c|}
\hline \multirow[b]{2}{*}{ Factors } & \multicolumn{4}{|c|}{ Singapore $(n=185)$} \\
\hline & Beta & $|t|$-statistics & $P$ value & VIF \\
\hline Constant & $12 \cdot 60$ & $9 \cdot 72$ & $<0.001 *$ & \\
\hline \multicolumn{5}{|l|}{ Demographic } \\
\hline - Religious belief ( 0 , no; 1 , yes) & $1 \cdot 12$ & $2 \cdot 60$ & $0 \cdot 010^{*}$ & $1 \cdot 001$ \\
\hline \multicolumn{5}{|l|}{ Attitude scores } \\
\hline $\begin{array}{l}\text { - Importance of hospital policy to } \\
\text { bereavement care }\end{array}$ & $0 \cdot 42$ & $3 \cdot 57$ & $<0.001 * *$ & $1 \cdot 716$ \\
\hline - Training for bereavement care & $0 \cdot 46$ & $5 \cdot 51$ & $<0.001 * *$ & $1 \cdot 714$ \\
\hline Adjusted $R^{2}$ & & $0 \cdot 42$ & & \\
\hline F-statistics & & $41 \cdot 83$ & $<0.001^{* *}$ & \\
\hline
\end{tabular}

Table 4 Multiple (stepwise) regression model on nurses and midwives' attitudes towards bereavement care by factors

Statistically significant at $* P<0.05 ; * P<0.01$.

VIF, variance inflation factor, a higher value meaning more dependent on other factors. 


\section{What is already known about this topic}

- When nurses or midwives provide bereavement care, negative attitudes may distract from the provision of good care, whilst positive attitudes can help bereaved couples to cope with the grieving process.

- Much research has been conducted to investigate the needs and feelings of bereaved families and the care practices that are helpful to them, but there is little mention of the needs and feelings of the nurses who care for them.

\section{What this paper adds}

- Nurses and midwives had a very positive attitude towards perinatal bereavement care.

- Almost all viewed their level of knowledge and understanding of grief counselling as insufficient and only $25 \cdot 5 \%$ had taken courses related to bereavement care.

- The goal of quality bereavement care can only be achieved when nurses' education and training needs are addressed.

\section{Implications for practice and/or policy}

- Nursing educators should include perinatal bereavement care in nursing or midwifery curricula and provide experience in clinical practice and this should be reinforced by staff development workshops in the hospitals.

- Nurses and midwives with appropriate education and skills should carry out perinatal bereavement care as part of their regular practice and appreciate the importance of referral when appropriate.

struggle (Hsu 1983). Infant loss violates the definition of a good death because it is coloured by early ending and physical deformation. Such cultural norms, which promote the importance of family continuity in achieving wholeness and good deaths, serves to punish mothers who fail to carry out their childbearing responsibilities. To our knowledge, no research has been carried out to recognize the experiences of Singaporean nurses/midwives, many of whom do not know the meaning of such rituals despite following them blindly; this especially concerns coping strategies and interpretation of feelings towards perinatal bereavement care in different ethnic groups. Thus, research in this area could be an important step towards understanding the bereavement process and opening up cross-cultural dialogue about it.
Students and junior nurses/midwives in the O\&G unit need the guidance of more experienced colleagues to be able to give effective bereavement care and learn strategies to avoid negative experiences. Mentoring by preceptors would provide a more educational and less stressful experience, increase the confidence and expertise of novices, and lead to increased quality of care for bereaved families.

For Singaporean nurses and midwives, religious belief was a factor affecting their attitudes towards bereavement care and this implies that education programmes about different religions and religious practices and referral opportunities should be considered. A systematic review conducted by Rowa-Dewar (2002) has shown no overall benefit from interventions among bereaved parents, except for highly distressed mothers whose psychological symptoms and marital dysfunction were statistically significantly reduced. Applied to practice, her findings suggest that some bereaved parents will benefit from a specific bereavement support programme. Therefore, undergraduate and ongoing education is necessary for staff, although in general most participants had a positive respect for bereaved parents' feelings and needs, and agreed that they should be given time to grieve. Respondents also believed that nurses working in the unit should be well- informed, with a clear policy for the management of bereavement, and given information that would help bereaved parents make plans and decisions for themselves. Some bereaved parents have felt that the care provided by nursing staff could also make them powerless (Paterson \& Zderad 1988; Chambers \& Chan 2000), and this phenomenon may occur when the focus is on symptoms or parts of the problem rather than on the whole person. Being treated as an object in this way can make parents keep their thoughts to themselves or lead them to feel that nursing staff were neglecting their thoughts, all of which may lead to insecurity and discouragement (Lundqvist et al. 2002).

\section{Conclusion}

Our findings support earlier reports that support and education are necessary to help nurses in their work with bereaved families. Bereavement counselling education and preceptorship supervision are recommended to reduce this stressful experience, increase the confidence and expertise of novices, and lead to increased quality of care for bereaved parents.

\section{Acknowledgement}

The authors would like to thank for the advices of Ms Paulin Koh, Assistant Director, Division of Nursing of the KK 
hospital, and the nurses/midwives in the KK hospital who volunteered to participate in this study.

\section{Funding}

This research received no specific grant from any funding agency in the public, commercial, or not-for-profit sectors.

\section{Conflict of interest}

No conflict of interest has been declared by the authors.

\section{Author contributions}

MFC was responsible for the study conception and design; performed the data analysis; and provided statistical expertise. DGA performed the data collection. MFC and DGA were responsible for the drafting of the manuscript; made critical revisions to the paper for important intellectual content.

\section{References}

Beem E.E., Eurelings-Bontekoe E.H.M., Cleiren M.P.H.D. \& Garssen B. (1998) Workshops to support the bereavement process. Patient Education and Counseling 34, 53-62.

Birtwistle J., Paynes S., Smith P. \& Kendrick T. (2002) The role of the district nurse in bereavement support. Journal of Advanced Nursing 38(5), 467-478.

Black D. \& Tufnell G. (2006) When is counseling indicated after a major traumatic life event? Current Paediatrics 16, 464-471.

Chambers H.M. \& Chan F.J. (2000) Support for Women/Families After Peri-Natal Death. (Cochrane Review), Issue 1. The Cochrane Library, Oxford. Update Software.

Chan C. \& Chow A. (1998) An indigenous psycho-educational counseling group for Chinese bereaved family members. Hong Kong Journal of Social Work 32(1), 1-26.

Chan M.F., Chan S.H. \& Day M.C. (2004) A pilot study on nurses' attitudes towards perinatal bereavement support: a cluster analysis. Nurse Education Today 24, 202-210.

Chan C.L.W., Chow A.Y.M., Ho S.M.Y., Tsui Y.K.Y., Tin A.F., Koo B.W.K. \& Koo E.W.K. (2005) The experience of Chinese bereaved persons: a preliminary study of meaning making and continuing bonds. Death Studies 29, 923-947.

Cox E. \& Briggs S. (2004) Disaster nursing: new frontiers for critical care. Critical Care Nurse 24(3), 16-22.

Engler A.J. \& Lasker J.N. (2000) Predictors of maternal grief in the year after a newborn death. Illness, Crisis \& Loss 8(3), 227243.

Engler A.J., Cusson R.M., Brockett R.T., Cannon-Heinrich C., Goldberg M.A., West M.G. \& Petow W. (2004) Neonatal staff and advanced practice nurses' perceptions of bereavement/end-of-life care of families of critically ill and/or dying infants. American Journal of Critical Care 13(6), 489-498.
Gao G., Ting-Toomey S. \& Gudykunst W.B. (1996) Chinese communication process. In The Handbook of Chinese Psychology (Bond M.H., ed.), Oxford University Press, Hong Kong, pp. 280293.

Gardner J.M. (1999) Perinatal death: uncovering the needs of midwives and nurses and exploring helpful interventions in the United States, England, and Japan. Journal of Transcultural Nursing 10, 120-130.

Gaze H. (2000) Breaking the silence. Community Practitioner 73(3), 504-505.

Gensch B.K. \& Midland D. (2000) When a baby dies: a standard of care. Illness, Crisis \& Loss 8, 286-295.

Gibson M. (1998) Order from Chaos. Responding to Traumatic Events, revised edn. Ventura Press, Birmingham.

Hsu F.L.K. (1983) Chinese kinship and Chinese behavior. In Rugged Individualism Reconsidered (Hsu F.L.K., ed.), University of Tennessee Press, Knoxville, pp. 246-389.

Hughes P., Turton P. \& Evans C. (1999) Stillbirth as a risk factor for depression and anxiety in subsequent pregnancies - a cohort study. British Medical Journal 318, 1721-1724.

Keeney G.B. (2004) Disaster preparedness: what do we do now? Journal of Midwifery \& Women's Health 49(4)Suppl. 1, 2-6.

Leininger M. (1997) Overview of the theory of culture care with the ethnonursing research method. Journal of Transcultural Nursing $8(2), 32-52$.

Lundqvist A., Nilstun T. \& Dykes A.K. (2002) Both empowered and powerless: mothers' experiences of professional care when their newborn dies. BIRTH 29(3), 192-199.

Martinson I.M. (1998) Funeral rituals in Taiwan and Korea. Oncology Nursing Forum 25(10), 1756-1760.

Paterson J.G. \& Zderad L.T. (1988) Humanistic Nursing, 2nd edn. National League for Nursing, New York.

Ping S.L., Chan Q.H.C. \& Lee T.F.D. (2002) Use of a bereavement service among suddenly bereaved familiers in Hong Kong. Journal of Clinical Nursing 11, 289-290.

Polit D.F. \& Hungler B.P. (1995) Nursing Research: Principles and Methods, 5th edn. Lippincott, Philadelphia, PA.

nQuery Advisor (2001) Statistical Solutions. nQuery Advisor, Belfast, North Ireland.

Robinson M., Baker L. \& Nackerud L. (1999) The relationship between attachment theory and perinatal loss. Death Studies 23, 257-270.

Rowa-Dewar N. (2002) Do interventions make a difference to bereaved parents? A systematic review of controlled studies. International Journal of Palliative Nursing 8(9), 452-457.

Rybarik F. (1996) What communications skills are most helpful with families grieving a perinatal loss? How can I express my concern while providing appropriate care? AWHONN Voice 4(6), 2.

Säflund K., Sjögren B. \& Wredling R. (2004) The role of caregivers after a stillbirth: views and experiences of parents. Birth 31(2), 132-137.

Speck P. (1978) Loss and Grief in Medicine. Balliere Tindall, London. Spiegel M.R. (1992) Theory and Problems of Probability and Statistics. McGraw-Hill, New York, pp. 112-113.

Stillbirth and Neonatal Death Society (SANDS) (1991) Miscarriage, Stillbirth and Neonatal Death: Guidelines for Professionals. SANDS, London.

Szgalsky J. (1989) Perinatal death, the family, and the role of the health professional. Neonatal Network 8(2), 15-19. 
White K., Coyne P. \& Patel U. (2001) Are nurses adequately prepared for end-of-life care? Journal of Nursing Scholarship 33, 147-151.

Wu D.Y.H. \& Tseng W.S. (1985) Introduction: the characteristics of Chinese culture. In Chinese culture and mental health
(Tseng W.S. \& Wu D.Y.H., eds), Academic Press, Orlando, pp. 3-13.

Yam B.M.C., Rossiter J.C. \& Cheung K.Y.S. (2001) Caring for dying infants: experiences of neonatal intensive care nurses in Hong Kong. Journal of Clinical Nursing 10, 651-659.

The Journal of Advanced Nursing (JAN) is an international, peer-reviewed, scientific journal. JAN contributes to the advancement of evidence-based nursing, midwifery and health care by disseminating high quality research and scholarship of contemporary relevance and with potential to advance knowledge for practice, education, management or policy. JAN publishes research reviews, original research reports and methodological and theoretical papers.

For further information, please visit the journal web-site: http://www.journalofadvancednursing.com 